\title{
Hígado graso no alcohólico: certezas e incertidumbres de una epidemia silenciosa
}

\author{
Esteban González Ballerga, ${ }^{1}$ Andrea Curia, ${ }^{1}$ Kenneth Cusi ${ }^{2}$ \\ ${ }^{1}$ División Gastroenterología, Sección Hepatología, Hospital de Clinicas José de San Martín, Universidad de Buenos Aires, Argentina. \\ ${ }^{2}$ División Endocrinología, Diabetes y Metabolismo, Universidad de Florida, Estados Unidos.
}

Acta Gastroenterol Latinoam 2020;50(3):236-252

\section{Resumen}

El hígado graso no alcohólico (HGNA) es la enfermedad hepática crónica más frecuente, con una prevalencia del 20-30\% en la población general y del 60-80\% en grupos de riesgo. Comprende un espectro de enfermedades que van de la esteatosis simple a la esteatohepatitis no alcohólica (EHNA), que puede evolucionar a diferentes grados de fibrosis hepática, cirrosis y todas sus complicaciones. Recientemente se ha sugerido un cambio en el nombre de esta entidad, y un grupo de expertos ha propuesto llamarla MAFLD, del inglés Metabolic (dysfunction) Associated Fatty Liver Disease. Este nombre aún no ha sido aceptado y ha generado muchas controversias. La esteatohepatitis no alcohólica se asocia a mortalidad cardiovascular, oncológica y hepatológica, por lo que requiere un enfoque multidisciplinario. Por el aumento de su prevalencia, el higado graso no alcohólico se ha convertido en un problema de salud relevante que requiere acciones especificas para su detección, diagnóstico, seguimiento y tratamiento. Identificar a los pacientes con riesgo de evolucionar a formas más severas es una tarea imprescindible, pero no sencilla. Los pacientes con esteatohepatitis, principalmente aquellos con fibrosis, caracteristica más importante asociada

Correspondencia: Esteban González Ballerga

Correo electrónico: eballerga@gmail.com a mortalidad, deberian ser identificados. El principal tratamiento para todos los estadios de la enfermedad consiste en las intervenciones sobre el estilo de vida y en la corrección agresiva de las comorbilidades. Otras terapias, como el tratamiento con pioglitazona o agonistas de la GLP-1, deberán ser seleccionadas y utilizadas en pacientes con esteatohepatitis no alcohólica con mayor riesgo de progresión de enfermedad hepática y cardiovascular, como son los pacientes obesos y diabéticos tipo 2.

Palabras claves. Higado graso no alcohólico, esteatosis, estetohepatitis, MAFLD, cirrosis, sindrome metabólico.

\section{Nonalcoholic fatty liver: certainties and uncertainties of a silent epidemic}

\section{Summary}

Nonalcoholic fatty liver disease (NAFLD) is the most frequent chronic liver disease, with an estimated prevalence of 20-30\% in the general population and increased to 60$80 \%$ in risk groups. It encompasses the spectrum of diseases ranging from simple steatosis to nonalcoholic steatohepatitis (NASH), which can progress to variable degrees of fibrosis, cirrhosis and all its complications. It is associated with cardiovascular, neoplasm and liver mortality, requiring a multidisciplinary approach. A change in the entity name has recently been suggested and a group of experts has proposed calling it Metabolic (dysfunction) Associated Fatty Liver Disease (MAFLD). This name has not yet been accepted and has generated significant controversy. Due to the increase in its prevalence, nonalcoholic fatty liver disease has become a relevant health problem that requires specific actions for its detection, diagnosis, monitoring and treatment. Identifying 
patients at risk of progressing to more severe forms is essential but not too easy. Patients with nonalcoholic steatohepatitis should be identified; mainly those with fibrosis, the most important characteristic associated with mortality. The main treatment for all stages of the disease consists of lifestyle interventions and aggressive correction of comorbidities. Other therapies, such as treatment with pioglitazone or GLP-1RA, should be selected and used in patients with nonalcoholic steatohepatitis who are at higher risk of progression of liver and cardiovascular disease, like obese and type 2 diabetic patients.

Key words. Nonalcoholic fatty liver disease, steatosis, steatohepatitis, MAFLD, cirrhosis, metabolic syndrome.

\section{Abreviaturas}

HGNA: Hígado graso no alcohólico.

ES: Esteatosis simple.

EHNA: Esteatohepatitis no alcohólica.

MAFLD: Metabolic (dysfunction) associated fatty liver disease.

NAFLD: Nonalcoholic fatty liver disease.

NAFL: Simple steatosis.

NASH: Nonalcoholic steatohepatitis.

IR: Insulino resistencia.

SM: Sindrome metabólico.

DM2: Diabetes mellitus tipo 2.

IMC: Indice de masa corporal.

HCC: Hepatocarcinoma.

EH: Esteatohepatitis.

AGNEs: Ácidos grasos no esterificados.

IL: Interlecina.

AST: Aspartato aminotransferasa.

ALT: Alanino aminotransferasa.

GGT: Gamma glutamil transpeptidasa.

RM: Resonancia magnética.

ACV: Accidente cerebro vascular.

NAFLD activity score: Non alcoholic fatty liver disease activity score.

VPN: Valor predictivo negativo.

VPP: Valor predictivo positivo.

ARFI: Impulso por imagen de fuerza de radiación acuistica. AUROC: Area under the receiver operating characteristic.

\section{Conceptos claves}

- El HGNA es la enfermedad hepática crónica más frecuente.

- No es una enfermedad benigna, es sistémica y progresiva.

- La principal causa de muerte en estos pacientes es la cardiovascular, seguida de cáncer no hepático, y en tercer lugar la causa hepática.
- La fibrosis hepática es la principal característica asociada a mortalidad.

- Diagnóstico clínico incluye: laboratorio (transaminasas), paneles (FIB-4), ecografía, elastografía y biopsia hepática.

- El tratamiento agresivo de las comorbilidades metabólicas y modificaciones en el estilo de vida son el pilar del tratamiento.

- Tratamientos farmacológicos: vitamina E (no diabéticos); pioglitazona y agonistas de GLP-1 en pacientes con o sin diabetes.

\section{Introducción}

El HGNA es la enfermedad hepática crónica más frecuente en el mundo y representará, junto con sus complicaciones, la próxima epidemia en hepatología. Con los avances en el tratamiento de otras enfermedades hepáticas crónicas, como las hepatitis virales, y el aumento a nivel global de la obesidad y el sedentarismo, se espera que prontamente sea la principal causa de trasplante hepático.

Su prevalencia está aumentando de forma alarmante en el mundo. Un metaanálisis reciente, que incluyó a más de ocho millones de individuos de veintidós países, estimó que la prevalencia global fue del $25 \%$ en la población adulta. ${ }^{1}$ En la Argentina no contamos con datos claros de incidencia y prevalencia de esta enfermedad.

En 1980, Ludwing describió esta entidad como la presencia de grasa hepática en ausencia de ingesta significativa de alcohol, ${ }^{2}$ instaurando el término con el que se la conoce todavía: "enfermedad por hígado graso no alcohólica”. Desde allí, se la definió como la presencia de esteatosis hepática por imágenes o histología, en ausencia de otras causas de enfermedad hepática crónica y de otras causas de esteatosis, como consumo de alcohol significativo, uso prolongado de medicación esteatogénica o desórdenes monogénicos hereditarios. ${ }^{3}$ Por lo tanto, el diagnóstico de HGNA fue clásicamente de exclusión.

El HGNA comprende un espectro de patologías que van desde la esteatosis simple (ES), una entidad relativamente benigna, hasta la EHNA, la cara más agresiva de este espectro, que conlleva riesgo de fibrosis hepática progresiva, cirrosis y todas sus complicaciones. La diferenciación de estas dos entidades es histológica. En la ES hay esteatosis predominantemente macrovesicular $\geq 5 \%$ sin injuria hepatocelular en forma de balonización, mientras que en la EHNA se evidencia, además de la esteatosis, inflamación lobulillar y balonización, con o sin fibrosis. ${ }^{4}$ En la actualidad se sabe que este proceso es dinámico. Los pacientes pueden fluctuar entre ES y EHNA por períodos cortos, y los que padecen EHNA pueden progresar a fibrosis de forma rápida o lenta. Además, la 
fibrosis -característica histológica más importante asociada a mortalidad hepática- puede incluso revertirse. ${ }^{5}$

Se considera al HGNA un reflejo de la insulinoresistencia (IR) sistémica. La mayoría de los pacientes tienen síndrome metabólico (SM), y presentan comorbilidades metabólicas como obesidad, diabetes mellitus tipo 2 (DM2), hipertensión arterial y dislipidemia; todos factores de riesgo de enfermedad cardiovascular. ${ }^{6} \mathrm{Sin}$ embargo, es importante aclarar que la causa raíz es la IR, que puede estar presente aun sin la clínica de síndrome metabólico.

El HGNA es una condición multisistémica y compromete órganos extrahepáticos. La principal causa de muerte es la enfermedad cardiovascular, seguida del cáncer no hepático. El tercer lugar como causa de muerte lo ocupa la enfermedad hepática.

Por lo tanto, el HGNA no es una enfermedad inocente, sino que es sistémica y progresiva, y como veremos en los próximos puntos, tiene un arsenal diagnóstico y terapéutico aún limitado.

\section{Factores de riesgo}

El HGNA es una entidad compleja, que se ve afectada por factores ambientales interrelacionados y por una predisposición genética. ${ }^{7}$

Se evidencia mayor prevalencia y mayor estadio de HGNA a mayor edad. ${ }^{8}$ El sexo masculino es un factor de riesgo y la prevalencia en hombres es dos veces mayor que en mujeres. ${ }^{9,10}$

La relación del HGNA con la etnia ha cambiado en el tiempo. Estudios realizados en los Estados Unidos sugerían que los hispanos tenían mayor prevalencia al compararlos con no hispanos blancos, mientras que afroamericanos no hispanos tenían menor prevalencia. ${ }^{11}$ Actualmente se sabe que estas diferencias étnicas tendrían relación con la variabilidad genética. ${ }^{12}$ Se han identificado varios genes asociados a HGNA. Los polimorfismos genéticos más estudiados son el rs58542926 en el gen TM6SF2 (transmembrane 6 super family member 2 ), ${ }^{13}$ y la variante rs738409 del gen PNPLA3 (patatin-like phospholipase domain containing protein3). ${ }^{14}$ Esta última es dos veces más frecuente en los hispanos que en los afroamericanos (40\% vs. 19\%). Estas pruebas no pueden recomendarse por el momento para su uso en la práctica clínica.

El estilo de vida tiene impacto en el desarrollo de esta entidad. Dietas con alto consumo de carne roja, granos refinados y bebidas azucaradas, así como el sedentarismo y el tabaquismo, se consideran factores de riesgo independientes para el desarrollo de esta enfermedad. ${ }^{15-17}$

Los componentes del SM son factores de riesgo para desarrollar HGNA. La prevalencia de las condiciones más frecuentemente asociadas a HGNA fueron estudiadas en el metaanálisis ya mencionado, ${ }^{1}$ evidenciando obesidad en $51,34 \%$ de los pacientes con HGNA (IC 95\%, 41,38-61,20), DM2 en 22,51\% (IC 95\%, $17,92-27,89$ ), dislipidemia en $69,16 \%$ (IC 95\%, 49,9183,46), hipertensión arterial en 39,34\% (IC 95\%, 33,1545,88 ) y $\mathrm{SM}$ en $42,54 \%$ (IC $95 \%, 30,06-56,05$ ). La incidencia de HGNA aumenta a mayor número de componentes del SM. ${ }^{18}$ Todo el espectro de la obesidad, desde el sobrepeso hasta la obesidad mórbida, se asocian con HGNA y se considera un determinante de mal pronóstico. En obesidad mórbida con indicación de cirugía bariátrica, la prevalencia de HGNA es superior al $80 \% .{ }^{19}$ Sin embargo, los individuos delgados no están exentos de desarrollar HGNA. ${ }^{20}$ En población asiática, el 6-20\% de los pacientes con HGNA no tienen sobrepeso ni obesidad ${ }^{19}$, ${ }^{21}$ y estos pacientes no tienen menor severidad histológica al compararlos con aquellos con HGNA y obesidad. ${ }^{22}$ Es importante el concepto de "salud metabólica" más allá de la definición de obesidad, dado que un individuo puede estar metabólicamente enfermo a pesar de tener un índice de masa corporal (IMC) normal. Estos pacientes delgados, pero metabólicamente enfermos, presentan un perfil metabólico desfavorable, que se caracteriza por altos niveles de insulino-resistencia, alta prevalencia de hipertensión arterial y un perfil lipídico e inflamatorio desfavorable, ${ }^{23}$ y pueden presentar acumulación de grasa ectópica, predominantemente visceral. ${ }^{24}$ Los pacientes con HGNA metabólicamente enfermos tienen mayor riesgo de enfermedad cardiovascular al compararlos con individuos metabólicamente sanos, en forma independiente del IMC. ${ }^{25}$ En Latinoamérica, el HGNA se encuentra íntimamente asociado a obesidad y su prevalencia en individuos delgados es menor que la descripta en población asiática. La DM2 se encuentra íntimamente relacionada al HGNA; más del 70\% de los pacientes con esta patología tienen HGNA. ${ }^{26}$ La DM2 es un determinante de alto riesgo respecto del desarrollo de formas más severas de la enfermedad, con progresión a EHNA, cirrosis y hepatocarcinoma (HCC). ${ }^{27,28}$

Otras condiciones, como apnea obstructiva del sueño, hipotiroidismo, hipopituitarismo, hiperuricemia, déficit de vitamina D y síndrome de ovario poliquístico, independientemente de la obesidad y resecciones pancreatoduodenales, también se consideran asociadas a HGNA. ${ }^{29}$

\section{Fisiopatología}

La fisiopatología del HGNA es aún incierta y está asociada con múltiples mecanismos que pueden causar progresión a cirrosis. La complejidad del problema 
es evidente dado que existen diferentes mecanismos que promueven esteatosis hepática, esteatohepatitis $(\mathrm{EH})$ y fibrosis. Por ejemplo, de los pacientes que tienen esteatosis, solamente un $20 \%$ a $30 \%$ desarrolla $\mathrm{EH}$, mientras que menos del $10 \%$ desarrolla fibrosis avanzada o cirrosis; ${ }^{1,4}$ aun en aquellos de alto riesgo con obesidad y DM2. Las limitaciones para desarrollar nuevos tratamientos radican en que la mayoría de los mecanismos moleculares estudiados pertenecen a modelos animales y no han sido confirmados, en su mayoría, en humanos. Los modelos animales de HGNA no son ideales, ya que su biología es altamente especie-dependiente y la generación de $\mathrm{EH}$ es en muchos aspectos diferente de la observada en humanos. ${ }^{30}$

\section{a. Esteatosis hepática e insulino-resistencia}

A pesar de los múltiples mecanismos posibles, existe consenso acerca de que la IR y la lipotoxicidad están interrelacionadas, y ambas son prominentes en pacientes con $\mathrm{EH}$ y/o fibrosis. ${ }^{31} \mathrm{La}$ IR es esencial en el desarrollo de la esteatosis observada en pacientes con obesidad, prediabetes o DM2, pero también en el desarrollo de EHNA en pacientes no-obesos. ${ }^{32}$

La insulina promueve la utilización de glucosa en músculo e hígado. También en tejido adiposo juega un rol clave, regulando la lipogénesis y la lipólisis, y determinando así los niveles de ácidos grasos no esterificados (AGNEs). La IR en tejido adiposo es por definición una disminución de la respuesta a la insulina para suprimir lipólisis. Esto lleva a un aumento crónico de los niveles plasmáticos de AGNEs, que se depositan en forma ectópica en el hígado, promoviendo la esteatosis. En la obesidad, el $70 \%$ de los ácidos grasos del hígado tienen su origen en tejido adiposo. ${ }^{29}$ Además, la IR, en el tejido adiposo, muscular y hepático, promueve la hipersecreción de insulina y disminuye su degradación hepática, causando hiperinsulinemia que activa la síntesis hepática de triglicéridos, y contribuye de esta manera aún más a la esteatosis. ${ }^{29}$ Este exceso de triglicéridos en el hepatocito promueve la acumulación de lípidos considerados tóxicos, como las ceramidas y los diacilgliceroles, los cuales exacerban la IR y estimulan los mecanismos inflamatorios.

\section{b. Inflamación lobulillar y "balonización"}

La inflamación crónica en la EHNA tiene una estrecha vinculación con la disfunción mitocondrial, aunque los mecanismos son posiblemente múltiples, e incluyen la disfunción del retículo endoplásmico, estrés oxidativo y activación de múltiples vías inflamatorias, como fue descripto en una reciente revisión. ${ }^{33}$ El deterioro mitocondrial lleva a un colapso de las vías de oxidación mitocondrial de ácidos grasos y contribuye a la acumulación de lípidos considerados tóxicos. Esto lleva a la necrosis (balonización) del hepatocito, inflamación lobulillar, activación y reclutamiento de las células de Kupffer y aumento de la secreción de colágeno por parte de las células estrelladas.

\section{c. Mecanismos de fibrosis hepática}

La inflamación crónica en la EHNA conduce mediante diversos mecanismos a la apoptosis del hepatocito con liberación de citoquinas tales como las IL 1, 2 y 18 y TNF- $\alpha$ y- $\beta .{ }^{34}$ Existen estímulos proinflamatorios en el contexto de IR que promueven la lipotoxicidad. Otros estímulos, como la hiperglucemia en DM2, promueven de forma coordinada tanto la fibrogénesis como la disminución de la degradación fisiológica del tejido conectivo que causan el desarrollo paulatino de cirrosis en pacientes con EHNA. ${ }^{35}$ Factores genéticos, como alteraciones de las membranas que determinan la transferencia de lípidos intracelulares, como la variante rs738409 del gen PNPLA3, también tienen un rol directo en el funcionamiento y activación de las células hepáticas estrelladas. ${ }^{36}$

Desde una perspectiva clínica, los factores asociados con progresión de fibrosis y cirrosis en general son el aumento de peso y la obesidad, la presencia de DM2 y la severidad de la fibrosis hepática al momento de diagnóstico. $^{1,4,7,8}$

\section{Historia natural}

La historia natural del HGNA es todavía incierta y son pocos los estudios realizados con buen diseño para determinar su pronóstico a largo plazo. Los estudios más antiguos al respecto, ${ }^{37-42}$ que ya reportaban que la obesidad y la DM2 eran factores de riesgo para fibrosis, se llevaron a cabo con un número limitado de pacientes, altamente seleccionados y con un tiempo de seguimiento corto.

La EHNA se asocia a mayor tasa de progresión de la fibrosis hepática que la ES, pero esta enfermedad es dinámica y los pacientes pueden evolucionar de una a la otra. En una revisión sistemática reciente, se analizaron once estudios con biopsias pareadas, seis años promedio entre ambas biopsias, que incluyeron a 411 pacientes con HGNA $^{43}$ y demostraron progresión de la fibrosis en el $34 \%$, estabilidad en el $43 \%$ y regresión en el $23 \%$ de los pacientes. Al analizar las tasas de progresión de la fibrosis por año, la revisión mencionada estimó que se requerirían 14,3 años (IC 95\%, 9,1-50) y 7,13 años (IC 95\%, 4,8$14,3)$ para progresar de un estadio al siguiente en pacientes con ES y EHNA, respectivamente. ${ }^{36}$

Predecir en qué momento y qué pacientes con HGNA desarrollarán complicaciones hepáticas es muy dificulto- 
so, pero se sabe que el grado de fibrosis hepática es el mayor determinante de estas complicaciones y que su presencia se asocia en forma significativa con la mortalidad global. ${ }^{41}, 44,45$ En un estudio con seguimiento medio de veinte años de 646 pacientes con diagnóstico de HGNA por biopsia, se evaluó el efecto de la presencia de EHNA y el estadio de fibrosis sobre la mortalidad global y hepática. ${ }^{43}$ Los pacientes con estadios de fibrosis $\geq$ F2 en la biopsia basal tuvieron mortalidad global aumentada con respecto a los controles, al compararlos con pacientes en estadios F0 y F1. El riesgo de desarrollar hepatopatía severa también fue significativamente superior en este grupo de pacientes al compararla con controles $(12 \%$ vs. $2 \%, p<0,001)$, con riesgo aumentado a partir del estadio de fibrosis F2 basal. ${ }^{43}$

Los pacientes con cirrosis por EHNA pueden desarrollar todas las complicaciones. El HGNA se está transformando, a nivel global, en una de las principales causas de HCC, relacionada con el aumento de la prevalencia de obesidad y DM2 en la población general. En la Argentina, el HGNA es la tercera causa de HCC, luego de la hepatitis $\mathrm{C}$ y de la enfermedad hepática por alcohol, con una incidencia en aumento, al igual que lo reportado en Europa y en los Estados Unidos. ${ }^{46} \mathrm{La}$ incidencia anual acumulada de HCC en la cirrosis por EHNA es del 2,6\%. ${ }^{47}$ En los últimos años se han descripto reportes de casos y series de HCC en HGNA sin cirrosis. ${ }^{48} \mathrm{La}$ incidencia de HCC en HGNA sin cirrosis es baja, < 1,5\%/año, por lo que no está indicada la vigilancia en pacientes sin fibrosis significativa.

\section{Nuevas definiciones y nuevas controversias}

Desde la primera definición de HGNA, este término y sus criterios diagnósticos no habían sido revisados. Recientemente, un grupo de expertos internacionales en consenso acordaron que el nombre de esta enfermedad no reflejaba el conocimiento que tenemos de su patogénesis, en la cual la disfunción metabólica sistémica tiene un rol central. Por este motivo, sugirieron llamarla "enfermedad por hígado graso asociada a disfunción metabólica" (metabolic dysfunction associated fatty liver disease [MAFLD] $).{ }^{49}$ A continuación exponemos la argumentación para esta nueva nomenclatura y definición, así como el debate que ha generado, y las razones por las que este cambio es considerado prematuro por muchos expertos. ${ }^{50}$

El diagnóstico de HGNA fue siempre de exclusión y para definirlo era mandatorio excluir otras causas de hepatopatía crónica, incluyendo el consumo de alcohol significativo. Este concepto teórico es dificultoso en la práctica cotidiana. La prevalencia creciente de esta enfermedad ha llevado a un aumento de casos en la vida real donde el HGNA coexiste con otras enfermedades hepáticas crónicas (HGNA con hepatitis virales, alcohol o enfermedades autoinmunes), lo que permite cuestionar la posibilidad de que su diagnóstico pueda seguir siendo de exclusión.

Los expertos plantearon definirla de forma más simple, por criterios positivos o de inclusión y de manera independiente al consumo de alcohol $\mathrm{u}$ a otras enfermedades hepáticas crónicas concomitantes.

Así, en este consenso, definen MAFLD como un trastorno metabólico con presencia de grasa hepática por imágenes, histología y/o biomarcadores séricos más uno de los tres siguientes: 1) sobrepeso/obesidad, 2) DM2, 3) evidencia de disregulación metabólica, para cuyo diagnóstico se requerirían al menos dos de las siguientes factores de riesgo metabólicos: circunferencia de cintu$\mathrm{ra} \geq 102 / 88 \mathrm{~cm}$ en hombres $\mathrm{y} \geq 90 / 80 \mathrm{~cm}$ en mujeres, prediabetes, inflamación con proteína $C$ reactiva elevada, hipertensión arterial, HDL disminuido, hipertrigliceridemia, $\mathrm{HOMA} \geq 2,5 .{ }^{51}$

En la última etapa de esta enfermedad, las características histológicas típicas de EHNA (esteatosis e inflamación) tienden a desaparecer. Es por esto que sugieren llamar cirrosis por $\mathrm{MAFLD}^{49}$ a aquella que no presenta los signos histológicos típicos de EHNA, en pacientes con factores de riesgo metabólicos y esteatosis, en biopsia hepática y/o en las imágenes previas.

Los pacientes que presentan otras hepatopatías crónicas y cumplen además criterios de MAFLD serían considerados por estos autores como MAFLD con patologías coexistentes. ${ }^{49}$ Por último, sugieren llamar causas alternativas de hígado graso a aquellas conocidas hasta la fecha como secundarias a esteatosis, que también era necesario excluir anteriormente para el diagnóstico de esta entidad. ${ }^{49}$

A pesar del valor de definir la enfermedad en términos positivos y de poner el énfasis en el componente metabólico, existe un número de razones válidas para considerar que el cambio es prematuro y que se soportaría mantener el término HGNA. ${ }^{48}$ Estas razones incluyen aspectos prácticos y científicos a la vez: a) recién ahora el término HGNA está siendo reconocido por médicos generales y por el público, por lo que un nuevo término podría causar confusión; b) la vaguedad del término metabólico para nombrar una enfermedad; c) la heterogénea biología del HGNA y la esperanza de poder llegar en el futuro a un nombre ligado a una fisiopatología más precisa; d) aspectos legales ligados a la aprobación de nuevas drogas que están siendo desarrolladas con el término actual y al reconocimiento de los entes respectivos; y e) promover el cambio con menos apresuramiento y con un consenso 
más amplio, implicando a todas las organizaciones regionales relacionadas al manejo del HGNA.

Sin duda, este debate es positivo y ayudará a reflexionar en el futuro sobre la complejidad del problema.

\section{Motivo de consulta habitual en hepatología}

El HGNA es una de las consultas más frecuentes en hepatología. El motivo de derivación al especialista es habitualmente hipertransaminasemia asintomática y/o hiperecogenicidad hepática por ecografía. ${ }^{52}$ Otros pacientes son derivados por hallazgos de SM. Los pacientes son asintomáticos y no suelen tener hallazgos al examen físico hasta que desarrollan estigmas de enfermedad hepática avanzada y/o signos o síntomas de descompensación hepática. El paciente habitual tiene sobrepeso u obesidad y adiposidad visceral con circunferencia de cintura aumentada. Los pacientes con IMC normal son infrecuentes. ${ }^{19}$

En el laboratorio pueden presentar elevación moderada de aspartato aminotransferasa (AST) y alanino aminotransferasa (ALT), con ALT característicamente mayor a AST. Sin embargo, más de la mitad de los pacientes con EHNA e incluso con cirrosis pueden tener transaminasas normales. ${ }^{53}$ Por lo tanto, la determinación aislada de transaminasas tiene una pobre correlación con la intensidad y el riesgo de progresión de la enfermedad. Elevaciones de la gamma glutamil traspeptidasa (GGT) son frecuentes y se han asociado a mayor fibrosis, mortalidad hepática y global. ${ }^{54,55}$ Es habitual evidenciar resistencia a la insulina, hiperglucemia, hipertrigliceridemia y descenso de colesterol HDL en el perfil lipídico, lo que se conoce como dislipemia aterogénica, y que tendría un rol central en el desarrollo de enfermedad cardiovascular. ${ }^{56}$ Los anticuerpos factor antinuclear y antimúsculo liso pueden estar presentes en hasta un $30 \%$ de los pacientes, ${ }^{57}$ en títulos bajos, y en algunas oportunidades es un desafío el diagnóstico diferencial con enfermedades autoinmunes. Elevaciones de ferritina se evidencian con frecuencia. El HGNA es la causa más frecuente de hiperferritinemia. El patrón típico en HGNA es valores de ferritina $<1000$ $\mathrm{mg} / \mathrm{L},{ }^{58}$ con ferremia y saturación de transferrina normales. Este aumento de ferritina no siempre se correlaciona con una sobrecarga de hierro. El 35-50\% de los pacientes presentan síndrome de sobrecarga de hierro dismetabólico, ${ }^{59,60}$ caracterizado por sobrecarga de hierro hepática y en otros depósitos corporales asociado con componentes del SM, en ausencia de otras causas identificables de exceso de hierro. ${ }^{61}$ Estos pacientes presentan saturación de transferrina normal o levemente elevada, con sobrecarga de hierro no mayor $150 \mathrm{mmol} / \mathrm{g}$ en resonancia magnética (RM) y un depósito moderado de hierro con distribución mixta, tanto en hepatocitos como en sistema reticuloendotelial en la biopsia hepática. ${ }^{62} \mathrm{El}$ impacto clínico del síndrome de sobrecarga de hierro dismetabólico no ha sido dilucidado y no pueden recomendarse flebotomías como tratamiento en estos pacientes. Excepcionalmente, el aumento de ferritina puede deberse a hemocromatosis hereditaria como enfermedad coexistente.

\section{Diagnóstico}

¿Tiene nuestro paciente hígado graso no alcohólico? ¿Tiene otra enfermedad hepática crónica coexistente?

El primer paso en el diagnóstico de HGNA es confirmar la presencia de esteatosis hepática. La ecografía es el método de elección. Es un método no invasivo con sensibilidad y especificidad (60-94\%, respectivamente). En pacientes con esteatosis leve $(<20 \%)$ la sensibilidad es baja, ${ }^{63}$ por lo que la ecografía confirma el diagnóstico de esteatosis, pero un resultado negativo no la descarta. La atenuación de parámetro controlado (CAP) y la espectroscopía por RM son métodos más sensibles para este fin. Esta última permite una estimación cuantitativa de la cantidad de grasa hepática. Sin embargo, estos métodos no están recomendados como primera opción por su alto costo y la falta de disponibilidad en la mayoría de los centros. ${ }^{64}$ Los biomarcadores séricos de esteatosis podrían ser útiles para predecir esteatosis con alta especificidad. El Hepatic Steatosis Index ${ }^{65}$ y el Fatty Liver Index (FLI) ${ }^{66}$ fueron validados independientemente y podrían ser de utilidad para el cribado de HGNA en población general y en pacientes de riesgo, como en DM2 u obesidad. ${ }^{3,67}$

Una vez hecho el diagnóstico de esteatosis debemos evaluar si existen causas alternativas o secundarias de hígado graso (Tabla 1). Las más frecuentes en adultos son el consumo significativo de alcohol y la exposición o consumo crónico de algunos fármacos. Por otro lado, debe excluirse la presencia de otras enfermedades hepáticas crónicas. En la Tabla 2 presentamos un listado con los parámetros que debemos estudiar en estos pacientes. Resaltamos la importancia de estudiar hepatitis virales, principalmente hepatitis $\mathrm{C}$, ya que entre el $50-80 \%$ de los infectados desconocen su condición y es altamente prevalente en DM2, así como también se recomienda aprovechar la oportunidad para vacunar a los susceptibles para Hepatitis A y B. La enfermedad hepática por alcohol es indistinguible histológicamente del HGNA. Se recomienda un interrogatorio minucioso para evaluar consumo de alcohol y realizar diagnóstico diferencial de estas dos patologías, o diagnosticarlas en forma coexistente. 
Tabla 1. Causas secundarias de hígado graso

- Consumo excesivo de alcohol.

- Fármacos: amiodarona, tamoxifeno, metotrexate, corticoides, ácido valproico, antirretrovirales.

- Hepatitis C (genotipo 3).

- Enfermedad de Wilson.

- Enfermedad celíaca.

- Nutrición parenteral.

- Deprivación calórica subaguda o crónica grave.

- Lipodistrofias.

- Síndrome de Reye.

- Esteatosis asociada al embarazo, síndrome de HELPP.

- Errores del metabolismo innatos: déficit de lipasa ácida lisosómica.

Tabla 2. Check list en pacientes con HGNA

- Enfermedad hepática por alcohol: > $30 \mathrm{gr} / d i ́ a$ en hombres, $>20$ gr/día mujeres.

- Hepatitis virales: Anti HBc, HBs Ag, anti HCV.

- Hepatopatías por tóxicos

- Hepatopatías autoinmunes: proteinograma electroforético, FAN, ASMA, AMA. *

- Hemocromatosis hereditaria: ferritina, ferremia, transferrina, saturación de transferrina.

- Enfermedad celíaca: IgA, Ac antitransglutaminasa IgA.

- Enfermedad tiroidea: TSH.

- Enfermedad de Wilson: ceruloplasmina, cupremia, cupruria.

- Déficit de alfa 1 antitripsina: dosaje alfa 1 antitripsina.

${ }^{\star}$ FAN: factor antinuclear; ASMA: anticuerpo antimúsculo liso; AMA: anticuerpo antimitocondrial.

$¿$ Tiene nuestro paciente comorbilidades asociadas al hígado graso no alcohólico?

Es mandatario evaluar cuidadosamente las comorbilidades más comúnmente asociadas a HGNA: obesidad, hipertensión arterial, dislipemia, DM2 o insulino-resisten- cia, hipotiroidismo, síndrome de ovario poliquístico, apnea obstructiva del sueño, entre otras. El trabajo multidisciplinario es indispensable en esta entidad multisistémica.

El HGNA es un factor de riesgo independiente de muchas enfermedades cardiovasculares (cardiopatía isquémica, miocardiopatías, arritmias y accidente cerebro vascular). ${ }^{27}$ Por otro lado, el HGNA se asocia con un riesgo aumentado de enfermedad renal crónica y esta se encuentra presente en el $20-50 \%$ de los pacientes con HGNA. ${ }^{68}$ Las neoplasias extrahepáticas se localizan tanto en el tracto gastrointestinal, principalmente colon y, con menos frecuencia, esófago, estómago y páncreas, como en localizaciones extraintestinales, riñón en hombres y mama en mujeres. ${ }^{69}$

\section{Métodos para establecer pronóstico}

¿Tiene nuestro paciente riesgo de hígado graso no alcohólico y fibrosis? ¿Cómo identificamos a los pacientes que tendrán mal pronóstico?

Para poder establecer el pronóstico hepático en HGNA es necesario determinar si el paciente tiene o no EHNA $y$, principalmente, conocer el estadio de fibrosis. Es bien conocido que la transición de ES a EHNA es un punto clave para la enfermedad hepática progresiva que lleva a cirrosis y HCC. ${ }^{70}$ Sin embargo, Eslam y col. ${ }^{47}$ cuestionan la utilidad de esta diferenciación dicotómica entre EHNA y ES en MAFLD y sugieren dejarla de lado, definiendo la severidad por el grado de actividad y el estadio de fibrosis, al igual que en otras enfermedades hepáticas crónicas. La cuantificación del grado de fibrosis es la característica más importante en la evaluación del riesgo en HGNA, ya que se relaciona con la mortalidad de causa hepática y global.

\section{a. Biopsia hepática}

Es el método considerado gold standard en HGNA. Permite diferenciar ES de EHNA, confirmar el diagnóstico, determinar el grado de actividad inflamatoria y el estadio de fibrosis y diagnosticar otras enfermedades coexistentes. Para el diagnóstico de HGNA se requiere la presencia de grasa en más del 5\% de los hepatocitos. Para EHNA, además, se requiere evidenciar daño hepatocitario (balonización, cuerpos de apoptosis, necrosis lítica) e inflamación lobulillar (típicamente en la zona 3 del acino). Una vez realizado el diagnóstico, hay que cuantificar la actividad y el grado de fibrosis mediante uno de los sistemas de clasificación histológica. En el año 2005, Kleiner y col. publicaron el sistema de puntuación del grupo NASH Clinical Research Network. ${ }^{71}$ Es el más utilizado en la actualidad y está compuesto por el NAFLD Activity Score (NAS) para clasificar la actividad de la lesión hepática y una clasificación del estadio de fibrosis. El NAS se 
obtiene sumando los scores individuales de esteatosis, balonización e inflamación lobular, con un rango total posible de 0 a 8 (Tabla 3). Si bien el NAS no es diagnóstico en sí mismo, sus valores se correlacionan con el diagnóstico histopatológico y valores de 0-2 se considerarían ES, mientras que puntajes $\geq 5$ se considerarían EHNA. Valores intermedios no fueron bien clasificados, por lo que no tendrían valor diagnóstico. La clasificación del estadio de fibrosis va desde su ausencia (estadio F0) a la cirrosis (estadio F4) (Tabla 3). Estos sistemas de puntuación son más útiles para la investigación que para el diagnóstico y su utilidad se basa en proporcionar una herramienta uniforme y reproducible para evaluar la gravedad de la enfermedad en ensayos clínicos, en estudios de historia natural y de evaluación de respuesta al tratamiento.

Tabla 3. Clasificación histológica de la actividad y estadio de fibrosis en HGNA (adaptado de Kleiner et al. 2005) ${ }^{69}$

\section{NAFLD Activity score (NAS) (0-8)}

Esteatosis (0-3)

$0:<5 \%$ hepatocitos comprometidos

1: $5-33 \%$ hepatocitos comprometidos

2: $33-66 \%$ hepatocitos comprometidos

3: $>66 \%$ hepatocitos comprometidos

Inflamación lobular (0-3)

0 : ninguna

$1:<2$ focos por campo óptico $\times 200$

2: 2-4 focos por campo óptico $\times 200$

3: > 4 focos por campo óptico $\times 200$

\section{Balonización (0-2)}

0 : ninguna

1: pocas células con balonización

2: muchas células con balonización

\section{Correlación entre el puntaje NAS y el diagnóstico histológico de EHNA}

$\geq 5$ : EHNA probable 0 definitiva

3-4: indeterminado

$\leq 2$ : no EHNA

\section{Puntuación de fibrosis}

1: fibrosis perisinusoidal o periportal

A: fibrosis leve perisinusoidal en zona 3

B: fibrosis moderada perisinusoidal en zona 3

C: fibrosis portal/periportal

2: fibrosis perisinusoidal en zona 3 con fibrosis portal/periportal

3: fibrosis en puente

4: cirrosis
La biopsia hepática es un procedimiento invasivo, con morbimortalidad asociada, variabilidad inter e intraobservador y errores de muestreo. ${ }^{72}$ Para reducir estas limitaciones es clave que la biopsia sea realizada por un hepatólogo con experiencia, que obtenga una muestra adecuada. Deben utilizarse agujas de 1,4-1,6 mm de diámetro (16 o $15 \mathrm{G}$ ), la muestra no debe medir menos de $1,6 \mathrm{~cm}$ de longitud y debe contar con al menos 10 espacios porta. Es fundamental que la muestra sea analizada por un patólogo experimentado. ${ }^{71}$

\section{b. Métodos no invasivos}

Al ser el HGNA una entidad de tan elevada prevalencia y la biopsia hepática un procedimiento no exento de complicaciones, es indispensable contar con métodos no invasivos. La estimación de fibrosis hepática también puede realizarse mediante scores clínico-bioquímicos o estudios por imágenes, y permiten identificar a los pacientes que se beneficiarían más con la realización de una biopsia hepática.

- Scores clínico-bioquímicos: el NAFLD Fibrosis Score (NFS) $)^{73}$ y la calculadora de fibrosis 4 (FIB-4) $)^{74}$ fueron validados externamente en HGNA. Solo requieren datos clínicos (IMC, edad) y/o bioquímicos (plaquetas, transaminasas, albúmina) habitualmente disponibles (Tabla 4). El score de BARD ${ }^{75}$ incluye IMC, cociente AST/ALT y la presencia de DM2 (Tabla 4). La fortaleza de estos scores radica en sus elevados valores predictivos negativos para descartar fibrosis avanzada, siendo sus valores predictivos positivos modestos, ${ }^{76-78}$ por lo que son útiles para descartar la presencia de fibrosis hepática avanzada.

- Métodos elastográficos: evalúan mediante diferentes técnicas de imagen la rigidez hepática, que se correlaciona con la fibrosis en el hígado. Existen diferentes métodos con una capacidad diagnóstica para evaluar fibrosis similar. Son estudios costosos y no están fácilmente disponibles en nuestro país.

Elastografía de transición controlada por vibración (FibroScan ${ }^{\circledR}$ ): es el método no invasivo más estudiado para evaluar fibrosis hepática. Permite mejores resultados para la detección de cirrosis que para el diagnóstico de fibrosis avanzada y tiene un VPN superior al VPP $(90 \%$ vs. $<65 \%) .{ }^{79}$ Es muy útil para descartar fibrosis significativa y para confirmar cirrosis, pero es menos precisa para definir estadios intermedios. Sus resultados son poco fiables en presencia de un IMC $>30 \mathrm{~kg} / \mathrm{m}^{2}$ y/o un grosor del pliegue torácico elevados. En una serie europea se obtuvieron resultados poco fiables en hasta un $20 \%$ de los estudios realizados, sobre todo en pacientes obesos. ${ }^{80}$ Para mejorar estos resultados y reducir el porcentaje de fallos, en obesidad se recomienda la utilización de sonda XL. ${ }^{77}$ 
Tabla 4. Scores clínico-bioquimicos para evaluación de fibrosis avanzada en HGNA

\begin{tabular}{|c|c|c|c|c|c|c|c|}
\hline Score & Componentes & Cálculo & Punto de corte & $\mathrm{n}$ & VPP (\%) & VPN (\%) & Ref. \\
\hline NFS & $\begin{array}{c}\text { AST/ALT, } \\
\text { GAA/DM, } \\
\text { edad, IMC, } \\
\text { plaquetas, } \\
\text { albúmina }\end{array}$ & $\begin{array}{c}-1,675+0,037 \times e d a d \\
\text { (años) + 0,094 x BMl + 1,13 } \\
\text { x ITG/DBT (si = 1, no = 0) + } \\
\text { 0,99 x AST/ALT - 0,013 x } \\
\text { plaquetas (x 109/l) - 0,66 x } \\
\text { albúmina (gr/dl) }\end{array}$ & $\begin{array}{c}<-1,455 \text { : excluye } \\
\text { FA } \\
>0,676 \text { : predice } \\
\text { FA }\end{array}$ & 733 & 82 & 88 & 71 \\
\hline FIB4 & $\begin{array}{l}\text { AST, ALT, } \\
\text { edad, } \\
\text { plaquetas }\end{array}$ & $\begin{array}{c}\text { (Edad [años] } \\
\text { AST[U/L])/(plaquetas [10 9] } \\
\sqrt{ } \text { ALT[U/L]) }\end{array}$ & $\begin{array}{c}<1,3 \text { : excluye FA } \\
>2,67 \text { : predice } \\
\text { FA }\end{array}$ & 541 & 80 & 90 & 76 \\
\hline BARD & $\begin{array}{l}\text { AST/ALT, } \\
\text { IMC, DM }\end{array}$ & $\begin{array}{c}\text { AST/ALT } \geq 0,8: 2 \text { puntos } \\
\text { IMC } \geq 28 \mathrm{Kg} / \mathrm{m}^{2}: 1 \text { punto } \\
\text { DM: } 1 \text { punto }\end{array}$ & $\begin{array}{l}<2 \text { : excluye FA } \\
\geq 2 \text { : predice FA }\end{array}$ & 827 & 43 & 96 & 73 \\
\hline
\end{tabular}

NFS: NAFLD fibrosis score; AST: aspartato aminotransferasa; ALT: alanino aminotransferasa; GAA: glucemia alterada en ayunas; DM: diabetes mellitus; IMC: índice de masa corporal; VPP: valor predictivo positivo; VPN: valor predictivo negativo; Ref.: referencias.

Elastosgrafía por onda de corte (SWE: Shear Wave Elastography): es una técnica de ultrasonido que evalúa la elasticidad hepática. El porcentaje de falla técnica y la tasa de resultados poco confiables son similares a los observados con FibroScan ${ }^{\circledR} .{ }^{81}$

Impulso por imagen de fuerza de radiación acústica (ARFI): también es una técnica de ultrasonido. En estudios en pacientes con HGNA se observó una buena precisión diagnóstica para fibrosis avanzada (AUROC 0,90). En estudios en HGNA, la tasa de falla técnica o de resultados poco confiables fue del $5,2 \%$, pero esas cifras ascienden al $28 \%$ en pacientes con IMC > $30 \mathrm{~kg} / \mathrm{m}^{2} .{ }^{82}$

Elastografía por RM: tiene mayor eficacia diagnóstica que los anteriores para la detección de fibrosis hepática avanzada $^{83}$ (AUROC 0,92-0,94), con una tasa de falla técnica del $7,7 \% .{ }^{84,85} \mathrm{Su}$ accesibilidad en nuestro país es mucho más limitada.

Valoración del paciente con "hígado graso no alcohólico": algoritmo diagnóstico

Al ser la fibrosis hepática el principal determinante de mortalidad, el diagnóstico de HGNA debe acompañarse siempre de la estadificación del grado de fibrosis.
La realización de biopsia hepática debe ser individualizada y no existe consenso respecto de las estrategias a utilizar en la práctica clínica para realizar solo aquellas indispensables. ${ }^{86,87}$ Aunque los métodos no invasivos son herramientas valiosas, debemos conocer muy bien sus limitaciones y considerarlos una ayuda para seleccionar pacientes que se beneficiarían al someterse a una biopsia hepática y no como reemplazo de esta.

Es indispensable en una enfermedad de tan alta prevalencia, en la que no se pueden realizar biopsias generalizadas, contar con un algoritmo para estratificar el riesgo. Muchos son los publicados y seguramente con los años aparecerán nuevos. En el año 2019, la AAEEH ${ }^{3}$ sugirió el que se muestra en la Figura 1, basándose en la combinación de métodos de imágenes y scores clínicobioquímicos que permitirían clasificar a los pacientes en riesgo bajo, intermedio o alto de fibrosis hepática. Este sistema permitiría identificar a un grupo de pacientes de muy bajo riesgo de fibrosis avanzada o cirrosis, en quienes se podría diferir la biopsia hepática, indicar tratamiento de las comorbilidades asociadas y prevenir las complicaciones cardiovasculares. En segundo lugar, permitiría identificar a un grupo de alto riesgo, en los que 
los parámetros clínico-bioquímicos (hipertensión portal, plaquetopenia) y la elastografía de transición evidencien alta sospecha clínica de fibrosis avanzada o cirrosis. Por último, un grupo de riesgo intermedio que se beneficiaría con una biopsia hepática, único medio para confir- mar la presencia de EHNA, fibrosis avanzada o cirrosis. En este algoritmo, se resalta la importancia de los scores clínico-bioquímicos, que podrían reducir la necesidad de biopsia al menos en el $75 \%$ de los casos, ${ }^{88}$ quedando a consideración en los grupos de riesgo alto e intermedio.

Figura 1. Algoritmo para la estratificación del riesgo en HGNA (adaptado de guía diagnóstico y tratamiento HGNA AAEEH 20193)
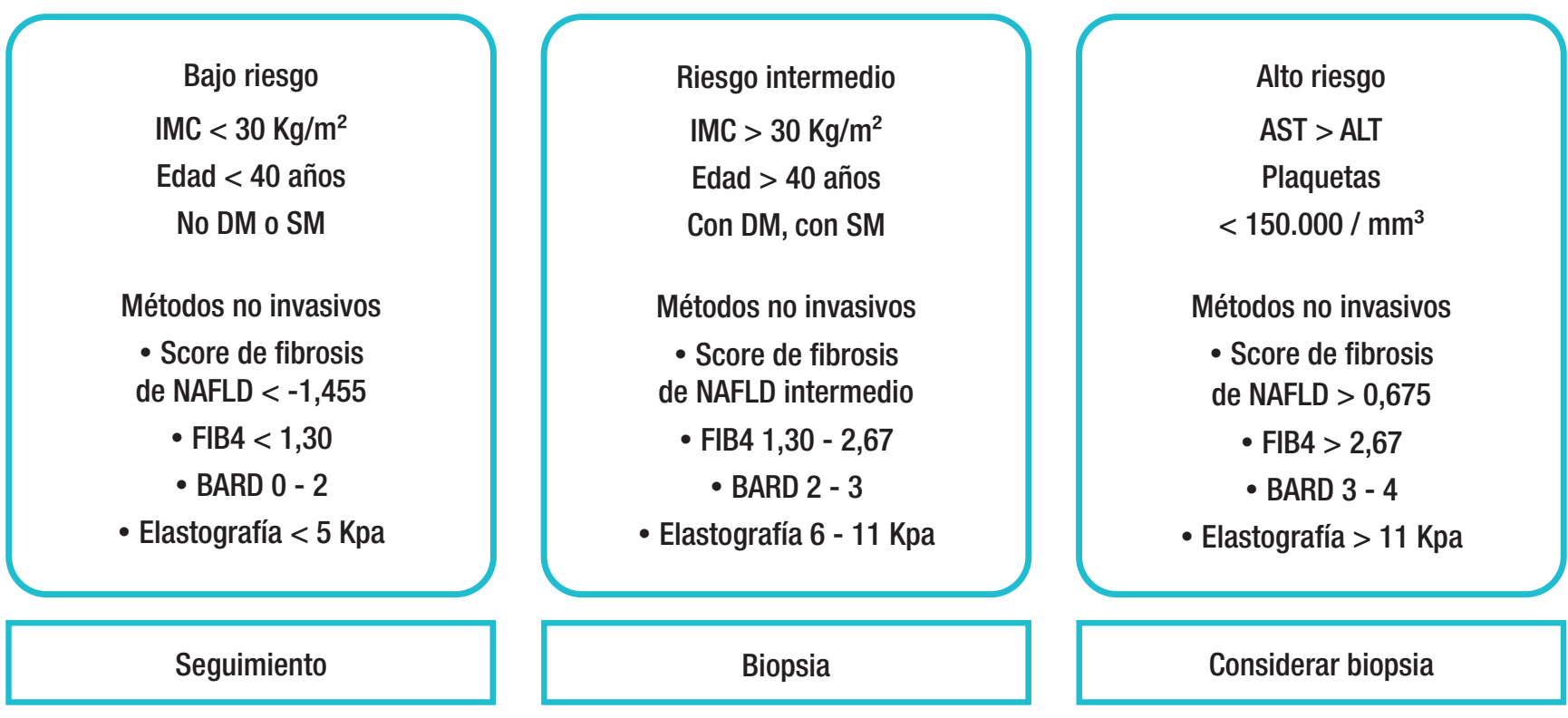

*IMC: índice de masa corporal; DM: diabetes mellitus; SM: síndrome metabólico.

\section{Cribado de hígado graso no alcohólico}

El cribado en HGNA es un tema controversial. Teniendo en cuenta la evidencia actual de que los pacientes con SM, obesidad y/o DM2 presentan mayor riesgo de enfermedad hepática severa, de morbimortalidad cardiovascular y de desarrollar neoplasias hepáticas y extrahepáticas, la AAEEH sugiere que en este grupo se lleve adelante. ${ }^{3}$ El método de elección para este fin es la ecografía hepática, por su disponibilidad en nuestro medio, por ser no invasivo, de bajo costo, sin riesgos y bien aceptado por los pacientes.

\section{Tratamiento}

El manejo óptimo del paciente con HGNA incluye tener en cuenta un número de factores: a) especial atención hacia aquellos con mayor riesgo de EHNA y fibrosis avanzada (pacientes con obesidad y síndrome metabólico, prediabetes o DM2, historia familiar de HGNA o cirrosis); b) establecer algoritmos para el diagnóstico temprano e implementarlos en forma sistemática; c) educar al paciente acerca de los riesgos serios relacionados con la presencia de HGNA y el valor de adoptar estilos de vida saludables; d) implementar un equipo multidisciplinario para el manejo de la enfermedad, tanto desde el punto de vista hepático como cardiometabólico; e) remover la desidia existente con respecto al tratamiento de EHNA con el uso de fármacos que han demostrado ser efectivos en estudios controlados y que se encuentran actualmente disponibles (vitamina E, pioglitazona, agonistas del receptor de GLP-1), y f) enfatizar el tratamiento de la obesidad con cambios en el estilo de vida, ejercicio, y cirugía bariátrica cuando esté indicada.

Es importante recalcar que el objetivo principal del manejo es identificar a los pacientes con esteatosis para llegar a identificar a aquellos con EHNA y fibrosis clínicamente significativa, y de esta manera, implementar tratamientos que reviertan, o por lo menos eviten, el progreso de la fibrosis hepática. El aspecto cardiometabólico es siempre importante, ya que los eventos cardiovasculares son la principal causa de mortalidad en HGNA, 
salvo cuando la presencia de fibrosis avanzada o cirrosis aumenta la mortalidad por causas de origen hepático. ${ }^{4}$

\section{Reducción del sobrepeso}

Hay consenso acerca de que el aspecto más relevante del manejo del paciente obeso con HGNA es inducir la pérdida de peso, revertir la IR y mejorar el perfil cardiometabólico desfavorable con una dieta hipocalórica y ejercicio. ${ }^{89-91}$ Numerosos estudios han demostrado su efectividad en mejorar la EHNA.92-94 En general, la mejoría de la EHNA es proporcional a la pérdida de peso y se cree que una pérdida $\geq 7 \%$ es necesaria en la mayoría de los pacientes. Sin embargo, es sorprendente que aún no haya estudios controlados de larga duración (mayor a 1 año) que comparen el impacto histológico de distintas modalidades de ejercicio o dietas hipocalóricas en pacientes con EHNA. La cirugía bariátrica es efectiva para revertir la EHNA en la mayoría de los pacientes. En cambio, el efecto sobre la fibrosis puede ser más variable, aunque en general también es proporcional a la pérdida de peso. ${ }^{95}$

\section{Agentes farmacológicos para el tratamiento de la diabetes mellitus 2}

Dada la fisiopatología del HGNA, no es de sorprender que los agentes más útiles hasta la fecha para revertir la EHNA son los que revierten la IR y la lipotoxicidad, ${ }^{87-89}$ ya sea cambiando la biología del tejido adiposo (pioglitazona), que vuelve a tener un funcionamiento semejante al tejido adiposo de un individuo de IMC normal e insulino-sensible, o bien reduciendo su efecto deletéreo mediante la pérdida de peso y de la masa de tejido adiposo enfermo (liraglutida, semaglutida). Es interesante que la metformina, que revierte la IR a nivel muscular y hepático pero no de forma tan significativa en tejido adiposo, no tiene un efecto beneficioso en la EHNA. ${ }^{88-90}$

\section{- Pioglitazona}

Desde el primer estudio controlado con pioglitazona en 2006, que sugería una significativa actividad en pacientes con prediabetes y DM2 con EHNA, ${ }^{96}$ varios estudios controlados de 12 a 36 meses de duración con casi 500 pacientes han demostrado de forma consistente su efectividad en pacientes $\operatorname{con}^{97,98}$ y $\sin$ DM2. ${ }^{95,96,99,}$ ${ }^{100}$ Induce resolución de la EHNA en un 40-50\% de los casos. En algunos estudios también se ha reportado una mejoría de la fibrosis hepática. ${ }^{95,97}$ La pioglitazona reduce la IR y mejora el metabolismo glucémico y lipídico, como agonista de los receptores nucleares PPAR gamma y alfa, muy abundantes en tejido adiposo. Ha sido el agente antidiabético más estudiado en pacientes con EHNA. Actualmente, la pioglitazona es aconsejada en varias guías para el manejo de pacientes con EHNA (AASLD 2019, ${ }^{4}$
EASL-EASD-EASO 2016, ${ }^{101}$ entre otras), siendo la droga antidiabética más efectiva. ${ }^{102}$ Es probable que su tratamiento transforme no solamente la historia natural de la enfermedad hepática, sino que también impacte en la mortalidad global de pacientes con EHNA, porque además disminuye la progresión de prediabetes a DM2. ${ }^{103}$ Asimismo, reduce eventos cardiovasculares en pacientes con diabetes, ${ }^{104-106} \mathrm{o}$ sin diabetes pero con IR. ${ }^{107}$ En un reciente análisis del estudio IRIS en pacientes que tomaron $\geq 80 \%$ de la medicación prescripta, ${ }^{108}$ la pioglitazona redujo un 36\% los ACV, un 53\% los síndromes coronarios agudos y un 39\% la combinación de ACV/infartos de miocardio/hospitalización por insuficiencia cardíaca. En contraste, la rosiglitazona no reduce eventos cardiovasculares y no es efectiva en la EHNA. ${ }^{88,89}$ Confirmando el rol de la IR y lipotoxicidad en la EHNA, es de interés observar que lanifibranor, un agonista PPAR alfa, gamma (como la pioglitazona) y delta, ha reportado recientemente en forma preliminar significativos beneficios en revertir la esteatohepatitis y fibrosis de pacientes con EHNA (NATIVE trial; www.ClinicalTrials.gov, ID: NCT NCT03008070).

El aumento de peso con pioglitazona es dosis-dependiente (en promedio 3,6 kilogramos) con dosis de 30 a 45 miligramos por día en estudios de 12-36 meses de duración. ${ }^{95-98}$ La dosis de 15 miligramos por día se asocia con mínimo aumento de peso $(1-2 \%)^{109}$ y actualmente se encuentra en estudio en pacientes con EHNA (www. ClinicalTrials.gov, ID: NCT04501406). Es importante destacar que el aumento de peso es por aumento de la grasa subcutánea, pero con una reducción de la grasa visceral (que es considerada aterogénica y asociada a riesgo cardiovascular), ${ }^{110}$ un factor que posiblemente contribuya a la disminución del riesgo cardiovascular observado con la pioglitazona. ${ }^{102-106}$ No debe ser prescripta a pacientes con historia de cáncer de vejiga, aunque en la mayoría de los estudios no se ha asociado a un aumento de su incidencia. ${ }^{87,88}$ Tampoco debe usarse si existe historia de insuficiencia cardíaca o edema preexistente de miembros inferiores (típicamente el desarrollo de edema ocurre en $5-8 \%$ de los pacientes con pioglitazona). La pioglitazona mejora la función ventricular en pacientes con DM2 sin falla ventricular, ${ }^{111}$ pero en presencia de disfunción ventricular por enfermedad coronaria, una potencial retención de líquidos puede precipitar insuficiencia cardíaca. ${ }^{102}$ Sin embargo, esto no ha sido evidenciado en estudios más recientes en los cuales existe mayor experiencia en la selección de pacientes. ${ }^{94-98,102-106}$ Debe monitorearse al paciente con osteoporosis porque puede aumentar la incidencia de fracturas, ${ }^{105}$ aunque se desconoce el mecanismo de acción de las tiazolidinedionas a nivel óseo. ${ }^{112}$ 
- Agonistas de los receptores de GLP-1

Esta es una clase de agentes cada vez más utilizados en el campo de la DM2 por sus beneficios no solo en reducir la hiperglucemia, sino también al conferir protección cardiovascular y renal. ${ }^{113} \mathrm{El}$ tratamiento se asocia con pérdida de peso de 6 al 16\% (dependiendo del agonista de GLP-1 usado), asociado a una reducción de las transaminasas y de la esteatosis hepática. Dado el efecto de los agonistas de los receptores de GLP-1 para inhibir el apetito e inducir pérdida de peso, no soprende que sea una clase de agente con propiedades promisorias para el tratamiento de la EHNA. La droga más estudiada es la liraglutida, y existen por lo menos once estudios publicados en la literatura reciente. ${ }^{114}$ En el único estudio randomizado, a doble-ciego, con biopsias hepáticas antes y después de 48 semanas de tratamiento comparado con placebo, la liraglutida mostró beneficios significativos con respecto a la EHNA y una reducción de la progresión de la fibrosis hepática. ${ }^{15}$ Esto es en contraste a la falta de eficacia clínica utilizando sitagliptina y otros inhibidores de DPP-IV en pacientes con HGNA. ${ }^{116}$ Recientemente, se reportaron resultados preliminares con semaglutida para el tratamiento de la EHNA (www.ClinicalTrials.gov ID NCT02970942). En un estudio de fase IIB se trataron 320 pacientes durante 72 semanas con semaglutide, a dosis diarias de 0,$1 ; 0,2$ y 0,4 miligramos, en el que un $62 \%$ tenía DM2. El resultado primario era la resolución de la EHNA sin empeoramiento de fibrosis. Semaglutida, en todas las dosis $(0,1 ; 0,2$ y $0,4 \mathrm{mg})$, redujo el peso corporal y mejoró la EHNA en el 40,4\% (0,1 mg), 35,6\% $(0,2 \mathrm{mg})$ y $58,9 \%(0,4 \mathrm{mg})$ de los pacientes, comparado a $17,2 \%$ con placebo. Estos prometedores resultados, junto con los beneficios cardiorrenales y metabólicos de esta clase de agentes, promete revolucionar el manejo de los pacientes con EHNA.

- Inhibidores de los cotransportadores de sodio-glucosa tipo 2

Este es un grupo creciente de agentes que también ha ganado significativa aplicación en pacientes con DM2 debido a sus beneficios cardiometabólicos, incluso respecto de la insuficiencia cardíaca e insuficiencia renal crónica. ${ }^{17}$ Estudios tempranos no controlados y una serie de estudios controlados recientemente publicados con dapagliflozina, ${ }^{118,119}$ canagliflozina ${ }^{120}$ y empagliflozina ${ }^{121}$ sugieren beneficio en pacientes con HGNA. Los resultados han sido consistentes, con una reducción moderada de peso, de las transaminasas y de la esteatosis hepática. ${ }^{122}$ Sin embargo, aún hay que aguardar para evaluar estudios que investiguen la histología hepática con este tratamiento.

En síntesis, los resultados obtenidos con medicacio- nes aprobadas para el uso en pacientes con DM2 abre la interesante posibilidad del tratamiento con "múltiple propósito", en donde se controle con un agente la glucemia y el perfil cardiometabólico, mientras se trata la EHNA. ${ }^{123,}{ }^{124}$ Se necesitan estudios a largo plazo para evaluar el impacto en la historia natural y la mortalidad hepática y extrahepática en esta población. Por último, es importante recordar que las guías de la $\mathrm{AASLD}^{4}$ apoyan el uso de las estatinas en pacientes con EHNA, dado su alto riesgo cardiovascular, aún con transaminasas moderadamente elevadas, pero esto requiere un monitoreo clínico adecuado.

\section{Vitamina E}

La vitamina $\mathrm{E}$ ha sido estudiada minuciosamente como prometedor agente antioxidante en pacientes con EHNA, aunque su mecanismo de acción es aún incierto. El estudio de mayor envergadura fue el PIVENS, ${ }^{96}$ que trató durante dos años a pacientes sin DM2 con EHNA con pioglitazona (30 miligramos por día), vitamina E (800 UI por día) o placebo. El estudio mostró eficacia clínica de la vitamina $\mathrm{E}$ versus placebo en $\mathrm{EH}$, pero la resolución de EHNA comparada con placebo fue solamente del $36 \%$ vs. $21 \%(p=0,05)$ y aparentemente menor que con pioglitazona ( $47 \%$ vs. $21 \% ; p=0,001$ vs. placebo), aunque una comparación directa entre estos tratamientos no fuera parte del diseño del estudio; tampoco hubo mejoría de la fibrosis. En un estudio pediátrico, la vitamina $\mathrm{E}$ tuvo un efecto modesto, mejorando la balonización pero no otros parámetros histológicos al compararse con placebo. ${ }^{125}$ Sin embargo, en un estudio observacional reciente, la vitamina $\mathrm{E}$ fue beneficiosa en pacientes adultos con EHNA y enfermedad hepática avanzada. ${ }^{126}$

En un estudio diseñado para evaluar el efecto aditivo de la pioglitazona y vitamina $\mathrm{E}$ en pacientes con DM2, la vitamina E como monoterapia redujo la esteatosis pero no otros parámetros histológicos, y no aumentó la respuesta a la pioglitazona. ${ }^{96}$ Se observó resolución de la EHNA en ambos grupos comparado con placebo (vitamina E como monoterapia: $33 \%$ vs. $12 \%, p=0,04$; combinando vitamina $\mathrm{E}$ con pioglitazona: $43 \%$ vs. $12 \%, p=0,005)$. Sin embargo, es posible que con un mayor número de pacientes hubiera sido significativo en mejorar EHNA, aunque es poco probable que lo fuera en mejorar la fibrosis. El temor con la vitamina $E$ es el potencial riesgo de aumento de la mortalidad observada en estudios epidemiológicos, ${ }^{127}$ del riesgo de $\mathrm{ACV}^{128}$ y de cáncer de próstata. ${ }^{129}$ Esto ha limitado su aceptación, pero la $\mathrm{AASLD}^{4}$ recomien- 
da su uso en pacientes sin diabetes o cirrosis si hay confirmación de EHNA con biopsia hepática.

\section{Trasplante hepático}

Hoy el HGNA es la segunda causa de trasplante hepático en el mundo y es la única indicación que está en aumento. Está indicado en cirrosis por EHNA en estadio avanzado y/o HCC que cumplan criterios de trasplantabilidad. Sin embargo, es menos probable que estos pacientes reciban un trasplante hepático y presenten mayor mortalidad en lista al compararlos con pacientes con cirrosis por hepatitis $\mathrm{C}$, alcohol, o ambas etiologías combinadas. ${ }^{130}$

En conclusión, el tratamiento en los pacientes con ES y EHNA sin fibrosis o fibrosis leve se basará en el abordaje global del estilo de vida, la optimización del tratamiento de las comorbilidades metabólicas asociadas, el control estrecho cardiovascular y el rastreo de cáncer no hepático. En cambio, los pacientes con EHNA con fibrosis moderada ( $\geq$ F2) deberían considerarse para tratamiento farmacológico además de las medidas ya mencionadas. No existe en la actualidad un tratamiento farmacológico aprobado; sin embargo, en los últimos años se ha avanzado mucho al respecto. Los pacientes con fibrosis avanzada y cirrosis tienen alto riesgo de complicaciones y mortalidad hepática. En ellos el foco del tratamiento está en intentar revertir el proceso fibrogénico y prevenir o tratar las complicaciones de la cirrosis. Deben seguir un esquema de vigilancia de HCC y de várices esofagogástricas según las recomendaciones habituales.

\section{Conclusiones}

El HGNA es una enfermedad pandémica, con múltiples mecanismos fisiopatológicos y una historia natural que desconocemos en profundidad, pero sabemos que no es benigna y que puede ser progresiva. Sus métodos diagnósticos son inciertos y tienen "zonas grises" que, a menudo, no nos permiten identificar a los pacientes de riesgo. El tratamiento universal, que es el descenso de peso, es altamente efectivo pero difícil. Los médicos debemos asumir la responsabilidad de que muchas veces fallamos en explicar la gravedad de la enfermedad y en ofrecer tratamientos multidisciplinarios para este fin. Sin embargo, también existen tratamientos farmacológicos que son poco utilizados, como la pioglitazona y los agonistas de GLP-1, que revierten la esteatohepatitis en aproximadamente el $50 \%$ de los pacientes y que, a la vez, pueden reducir su alto riesgo cardiovascular. El desarrollo de fármacos para la EHNA se encuentra en amplia expansión y hay varios fármacos en fases II y III con resultados publicados que demuestran efectos sobre la histología de la EHNA.
El cambio de nomenclatura a MAFLD acercaría el hígado graso a su fisiopatología, y la nueva definición consideraría la posibilidad de coexistir con otras patologías hepáticas crónicas, reconociendo la heterogeneidad de la enfermedad. Aunque es claro que se necesita un cambio, por el momento volver a clasificarla parece prematuro y esperamos un consenso que involucre a todas las partes interesadas antes de un cambio definitivo. Esto solo debe ser un primer paso y debería sentar las bases para seguir nuevas direcciones de investigación. La historia del hígado graso recién empieza a escribirse.

\section{Referencias}

1. Younossi ZM, Koenig AB, Abdelatif D, Fazel Y, Henry L, Wymer M. Global epidemiology of nonalcoholic fatty liver diseasemeta-analytic assessment of prevalence, incidence, and outcomes. Hepatology 2016; 64: 73-84.

2. Ludwig J, Viggiano TR, McGill DB, et al. Nonalcoholic steatohepatitis: Mayo Clinic experiences with a hitherto unnamed disease. Mayo Clin Proc 1980; 55: 434-438.

3. Fassio E, Dirchwolf M, Barreyro FJ. Primera guía de diagnóstico y tratamiento del hígado graso no alcohólico de la Asociación Argentina para el Estudio de las Enfermedades del Hígado. Asociación Argentina para el Estudio de las Enfermedades del Hígado, 2019. Disponible en: http://www.aaeeh.org.ar/es/attachment/show/40

4. Chalasani N, Younossi Z, Lavine J, Charlton M, Cusi K, Rinella M, Harrison S, Brunt E, Sanyal A. The diagnosis and management of nonalcoholic fatty liver disease: Practice guidance from the American Association for the Study of Liver Diseases. Hepatology 2018; 67: 328-357.

5. Tilg H, Effenberger M. From NAFLD to MAFLD: when pathophysiology succeeds. Nat Rev Gastroenterol Hepatol 2020; 17 (7): 387-388. doi:10.1038/s41575-020-0316-6.

6. Vernon G, Baranova A, Younossi ZM. Systematic review: the epidemiology and natural history of non-alcoholic fatty liver disease and non-alcoholic steatohepatitis in adults. Aliment Pharmacol Ther 2011; 34: 274-285.

7. De Oliveira CPMS, Cotrim HP, Arrese M. Factores de riesgo de la enfermedad por hígado graso no alcohólico en poblaciones de Latinoamérica: situación actual y perspectivas. Clin Liver Dis 2019; 13 (1): 5-8. doi: 10.1002/cld.837.

8. Koehler EM, Schouten JN, Hansen BE, van Rooij FJ, Hofman A, Stricker BH, et al. Prevalence and risk factors of nonalcoholic fatty liver disease in the elderly: results from the Rotterdam study. J Hepatol 2012; 57: 1305-1311.

9. Zelber-Sagi S, Nitzan-Kaluski D, Halpern Z, Oren R. Prevalence of primary non-alcoholic fatty liver disease in a populationbased study and its association with biochemical and anthropometric measures. Liver Int 2006; 26: 856-863.

10. Fattahi MR, Niknam R, Safarpour A, Sepehrimanesh M, Lotfi M. The prevalence of metabolic syndrome in non-alcoholic fatty liver disease; a population-based study. Middle East J Dig Dis 2016; 8: 131-137.

11. Masuoka HC, Chalasani N. Nonalcoholic fatty liver disease: an emerging threat to obese and diabetic individuals. Ann N Y Acad Sci 2013; 1281: 106-122. 
12. Dongiovanni P, Anstee QM, Valenti L. Genetic predisposition in NAFLD and NASH: impact on severity of liver disease and response to treatment. Curr Pharm Des 2013; 19: 5219-5238.

13. Kozlitina J, Smagris E, Stender S, et al. Exome-wide association study identifies a TM6SF2 variant that confers susceptibility to nonalcoholic fatty liver disease. Nat Genet 2014; 46: 352-356.

14. Romeo S, Kozlitina J, Xing C, et al. Genetic variation in PNPLA3 confers susceptibility to nonalcoholic fatty liver disease. Nat Genet 2008; 40: 1461-1465.

15. Satapathy SK, Sanyal AJ. Epidemiology and Natural History of Nonalcoholic Fatty Liver Disease. Semin Liver Dis 2015; 35: 221-235. [PMID: 26378640 DOI: 10.1055/s-00351562943]

16. Hamabe A, Uto H, Imamura Y, Kusano K, Mawatari S, Kumagai K, Kure T, Tamai T, Moriuchi A, Sakiyama T, Oketani M, Ido A, Tsubouchi H. Impact of cigarette smoking on onset of nonalcoholic fatty liver disease over a 10-year period. J Gastroenterol 2011; 46: 769-778. [PMID: 21302121 DOI: 10.1007/s00535011-0376-z]

17. Targher G, Alberiche M, Zenere MB, Bonadonna RC, Muggeo $\mathrm{M}$, Bonora E. Cigarette smoking and insulin resistance in patients with noninsulin-dependent diabetes mellitus. J Clin Endocrinol Metab 1997; 82: 3619-3624. [PMID: 9360516 DOI: 10.1210/ jcem.82.11.4351]

18. Kanwar P, Kowdley KV. The metabolic syndrome and its influence on nonalcoholic steatohepatitis. Clin Liver Dis 2016; 20: 225243. [PMID: 27063266 DOI: 10.1016/j.cld.2015.10.002]

19. Subichin M, Clanton J, Makuszewski M, Bohon A, Zografakis JG, Dan A. Liver disease in the morbidly obese: a review of 1000 consecutive patients undergoing weight loss surgery. Surg Obes Relat Dis 2015; 11: 137-141.

20. Chen F, Esmaili S, Rogers G, Bugianesi E, Petta S, Marchesini G, et al. Lean NAFLD: A distinct entity shaped by differential metabolic adaptation. Hepatology 2020; 71 (4): 1213-1227. doi:10.1002/hep.30908

21. Younossi Z, Anstee QM, Marietti M, Hardy T, Henry L, Eslam $\mathrm{M}$, et al. Global burden of NAFLD and NASH: trends, predictions, risk factors and prevention. Nat Rev Gastroenterol Hepatol 2018; 15 (1): 11-20. doi:10.1038/nrgastro.2017.109

22. Rastogi A, Shasthry SM, Agarwal A, Bihari C, Jain P, Jindal A, et al. Non-alcoholic fatty liver disease - histological scoring systems: a large cohort single-center, evaluation study. APMIS 2017; 125: 962-973.

23. Hinnouho MG, Czernichow S. Metabolically Healthy Obesity and Risk of Mortality Does the definition of metabolic health matter? Diabetes Care 2013; 36 (8): 2294-2300.

24. Stefan N, Schick F, Haring HU. Causes, characteristics, and consequences of metabolically unhealthy normal weight in humans. Cell Metab 2017; 26: 292-300.

25. Lassale C, Tzoulaki I, Moons KGM, Sweeting M, Boer J, Johnson $\mathrm{L}$, et al. Separate and combined associations of obesity and metabolic health with coronary heart disease: a pan-European casecohort analysis. Eur Heart J 2018; 39: 397-406.

26. Arrese M, Barrera F, Triantafilo N, Arab JP. Concurrent nonalcoholic fatty liver disease and type 2 diabetes: diagnostic and therapeutic considerations. Expert Rev Gastroenterol Hepatol 2019; 13: 849-866.

27. Anstee QM, Targher G, Day CP. Progression of NAFLD to diabetes mellitus, cardiovascular disease or cirrhosis. Nat Rev Gastroenterol Hepatol 2013; 10: 330-344.
28. Byrne CD, Targher G. NAFLD: a multisystem disease. J Hepatol 2015; 62: S47-S64.

29. VanWagner LB, Rinella ME. Extrahepatic manifestations of nonalcoholic fatty liver disease. Curr Hepatol Rep 2016; 15: 7585.

30. Van Herck MA, Vonghia L, Francque SM. Animal models of nonalcoholic fatty liver disease - a starter's guide. Nutrients 2017; 9: 1072 .

31. Cusi K. Role of obesity and lipotoxicity in the development of nonalcoholic steatohepatitis: Pathophysiology and clinical implications. Gastroenterology 2012; 142: 711-725.

32. Cusi K. Nonalcoholic steatohepatitis in non-obese patients: Not so different after all. Hepatology 2017; 65: 4-7.

33. Sunny NE, Bril F, Cusi K. Mitochondrial adaptation in nonalcoholic fatty liver disease: novel mechanisms and treatment strategies. Trends Endocrinol Metab 2017; 28: 250-260.

34. Allaire M, Rautou PE, Codogno P, Lotersztajn S. Autophagy in liver diseases: Time for translation? J Hepatol 2019; 70: 985-998.

35. Schuppan D, Surabattula R, Wang XY. Determinants of fibrosis progression and regression in NASH. J Hepatol 2018; 68: 238-250.

36. Lindén D, Ahnmark A, Pingitore P, et al. Pnpla3 silencing with antisense oligonucleotides ameliorates nonalcoholic steatohepatitis and fibrosis in Pnpla3 I148M knock-in mice. Mol Metab 2019; 22: 49-61.

37. Lee RG. Nonalcoholic steatohepatitis: a study of 49 patients. Hum Pathol 1989; 20 (6): 594-598.

38. Powell EE, Cooksley WG, Hanson R, Searle J, Halliday JW, Powell LW. The natural history of nonalcoholic steatohepatitis: a follow-up study of forty-two patients for up to 21 years. Hepatology 1990; 11 (1): 74-80.

39. Teli MR, James OF, Burt AD, Bennett MK, Day CP. The natural history of nonalcoholic fatty liver: a follow-up study. Hepatology 1995; 22 (6): 1714-1719.

40. Matteoni CA, Younossi ZM, Gramlich T, Boparai N, Liu YC, McCullough AJ. Nonalcoholic fatty liver disease: a spectrum of clinical and pathological severity. Gastroenterology 1999; 116 (6): 1413-1419.

41. Dam-Larsen S, Becker U, Franzmann MB, Larsen K, Christoffersen P, Bendtsen F. Final results of a long-term, clinical follow-up in fatty liver patients. Scand J Gastroenterol 2009; 44 (10): 1236-1243. Disponible en: https://doi.org/10.1080/ 00365520903171284.

42. Evans CD, Oien KA, MacSween RN, Mills PR. Non-alcoholic steatohepatitis: a common cause of progressive chronic liver injury? J Clin Pathol 2002; 55 (9): 689-692.

43. Singh S, Allen AM, Wang Z, et al. Fibrosis progression in nonalcoholic fatty liver vs nonalcoholic steatohepatitis: a systematic review and meta-analysis of paired-biopsy studies. Clin Gastroenterol Hepatol 2015; 13: 643-654.

44. Ekstedt M, Hagström H, Nasr P, et al. Fibrosis stage is the strongest predictor for disease specific mortality in NAFLD after up to 33 years of follow-up. Hepatology 2015; 61: 1547-1554.

45. Hagström H, Nasr P, Ekstedt M, et al. Fibrosis stage but not NASH predicts mortality and time to development of severe liver disease in biopsy-proven NAFLD. J Hepatol 2017; 67: 1265-1273.

46. Piñero F, Pages J, Marciano S, et al. Fatty liver disease, an emerging etiology of hepatocellular carcinoma in Argentina. World J Hepatol 2018; 10 (1): 41-50. doi:10.4254/wjh.v10.i1.41

47. Ascha MS, Hanouneh IA, Lopez R, et al. The incidence and risk factors of hepatocellular carcinoma in patients with nonalcoholic steatohepatitis. Hepatology 2010; 51: 1972-1978. 
48. Baffy G, Brunt EM, Caldwell SH. Hepatocellular carcinoma in non-alcoholic fatty liver disease: an emerging menace. J Hepatol 2012; 56 (6): 1384-1391. doi:10.1016/j.jhep.2011.10.027

49. Eslam M, Sanyal AJ, George J. International consensus panel MAFLD: A consensus-driven proposed nomenclature for metabolic associated fatty liver disease. Gastroenterology. Disponible en: https://doi.org/ 10.1053/j.gastro.2019.11.312 (2020).

50. Younossi ZM, Rinella ME, Sanyal A, Harrison SA, Brunt E, Goodman Z, Cohen DE, Loomba R. From NAFLD to MAFLD: Implications of a premature change in terminology. Hepatology 2020. Accepted Author Manuscript. doi:10.1002/hep.31420.

51. Eslam M, et al. A new definition for metabolic associated fatty liver disease: an international expert consensus statement. J Hepatol 2020. Disponible en: https://doi.org/ 10.1016/j. jhep.2020.03.039.

52. Patel V, Sanyal AJ, Sterling R. Clinical presentation and patient evaluation in nonalcoholic fatty liver disease. Clin Liver Dis 2016; 20: 277-292. [PMID: 27063269 DOI: 10.1016/j. cld.2015.10.006]

53. Fracanzani AL, Valenti L, Bugianesi E, et al. Risk of severe liver disease in nonalcoholic fatty liver disease with normal aminotransferase levels: a role for insulin resistance and diabetes. Hepatology 2008; 48: 792-798.

54. Ruhl C, Everhart J. Elevated serum alanine aminotransferase and $\mathrm{V}$-glutamyltransferase and mortality in the United States population. Gastroenterology 2009; 136: 477-485. doi.org/10.1053/j. gastro.2008.10.052

55. Tahan V, Canbakan B, Balci H, et al. Serum gamma-glutamyltranspeptidase distinguishes non-alcoholic fatty liver disease at high risk. Hepato-gastroenterology 2008; 55 (85): 1433-1438.

56. Bril F, Lomonaco R, Cusi K. The challenge of managing dyslipidemia in patients with nonalcoholic fatty liver disease. Clin Lipidol 2012; 7: 471-481.

57. Neuschwander-Tetri BA, Clark JM, Bass NM, Van Natta ML, Unalp-Arida A, Tonascia J, Zein CO, Brunt EM, Kleiner DE, McCullough AJ, Sanyal AJ, Diehl AM, Lavine JE, Chalasani N, Kowdley KV. Clinical, laboratory and histological associations in adults with nonalcoholic fatty liver disease. Hepatology 2010; 52 : 913-924. doi:10.1002/hep.23784

58. Mendler MH, Turlin B, Moirand R, et al. Insulin resistanceassociated hepatic iron overload. Gastroenterology 1999; 117: 1155-1163.

59. Valenti L, Fracanzani AL, Bugianesi E, et al. HFE genotype, parenchymal iron accumulation, and liver fibrosis in patients with nonalcoholic fatty liver disease. Gastroenterology 2010; 138: 905-912.

60. Nelson JE, Wilson L, Brunt EM, et al. Relationship between the pattern of hepatic iron deposition and histological severity in nonalcoholic fatty liver disease. Hepatology 2011; 53: 448-457.

61. Jezequel C, Laine F, Laviolle B, et al. Both hepatic and body iron stores are increased in dysmetabolic iron overload syndrome. A casecontrol study. Plos One 2015; 10: e0128530.

62. Turlin B, Mendler MH, Moirand R, et al. Histologic features of the liver in insulin resistance-associated iron overload. A study of 139 patients. Am J Clin Pathol 2001; 116: 263-270.

63. Festi D, Schiumerini R, Marasco G, Scaioli E, Pasqui F, Colecchia A. Non-invasive diagnostic approach to non-alcoholic fatty liver disease: current evidence and future perspectives. Expert Rev Gastroenterol Hepatol 2015; 9: 1039-1053. doi:10.1586/174741 24.2015.1049155
64. Rinella ME. Nonalcoholic fatty liver disease a systematic review. JAMA 2015; 313: 2263-2273. doi:10.1001/jama.2015.5370

65. Meffert PJ, Baumeister SE, Lerch MM, Mayerle J, Kratzer W, Volzke H. Development, external validation, and comparative assessment of a new diagnostic score for hepatic steatosis. Am J Gastroenterol 2014; 109: 1404-1014. doi:10.1038/ajg.2014.155

66. Bedogni G, Bellentani S, Miglioli L, Masutti F, Passalacqua M, Castiglione A, et al. The fatty liver index: a simple and accurate predictor of hepatic steatosis in the general population. BMC Gastroenterol 2006; 6: 33. doi:10.1186/1471-230X-6-33

67. Corey KE, Klebanoff MJ, Tramontano AC, Chung RT, Hur C. Screening for nonalcoholic steatohepatitis in Individuals with type 2 diabetes: A cost-effectiveness analysis. Dig Dis Sci 2016; 61: 2108-2117. doi:10.1007/s10620-016-4044-2

68. Musso G, Gambino R, Tabibian JH, et al. Association of nonalcoholic fatty liver disease with chronic kidney disease: a systematic review and meta-analysis. PloS Med 2014: 11: e1001680.

69. Sanna C, Rosso C, Marietti M, Bugianesi E. Non-alcoholic fatty liver disease and extra-hepatic cancers. Int J Mol Sci 2016; 17: 717-730.

70. Farrell GC, Larter CZ. Nonalcoholic fatty liver disease: From steatosis to cirrhosis. Hepatology 2006; 43: 99-112. doi:10.1002/ hep. 20973

71. Kleiner DE, Brunt EM, Van Natta M, Behling C, Contos MJ, Cummings OW, et al. Design and validation of a histological scoring system for nonalcoholic fatty liver disease. Hepatology 2005; 41: 1313-1321.

72. Bedossa P. Diagnosis of non-alcoholic fatty liver disease/non-alcoholic steatohepatitis: Why liver biopsy is essential. Liver International 2018; 38 (Suppl. 1): 64-66.

73. Angulo P, Hui JM, Marchesini G, Bugianesi E, George J, Farrell GC, et al. The NAFLD fibrosis score: a noninvasive system that identifies liver fibrosis in patients with NAFLD. Hepatology 2007; 45: 846-854. doi:10.1002/hep.21496

74. Sterling RK, Lissen E, Clumeck N, Sola R, Correa MC, Montaner J, et al. Development of a simple noninvasive index to predict significant fibrosis in patients with HIV/HCV coinfection. Hepatology 2006; 43: 1317-1325. doi:10.1002/hep.21178

75. Harrison SA, Oliver D, Arnold HL, et al. Development and validation of a simple NAFLD clinical scoring system for identifying patients without advanced disease. Gut 2008; 57 (10): 1441-1447.

76. Guha IN, Parkes J, Roderick P, Chattopadhyay D, Cross R, Harris $S$, et al. Noninvasive markers of fibrosis in nonalcoholic fatty liver disease: Validating the European Liver Fibrosis Panel and exploring simple markers. Hepatology 2008; 47: 455-460. doi:10.1002/hep.21984

77. McPherson S, Anstee QM, Henderson E, Day CP, Burt $\mathrm{AD}$. Are simple noninvasive scoring systems for fibrosis reliable in patients with NAFLD and normal ALT levels? Eur J Gastroenterol Hepatol 2013; 25: 652-658. doi:10.1097/ MEG.0b013e32835d72cf

78. Shah AG, Lydecker A, Murray K, et al. Comparison of noninvasive markers of fibrosis in patients with nonalcoholic fatty liver disease. Clin Gastroenterol Hepatol. 2009; 7 (10): 1104.

79. Wong VW-S, Vergniol J, Wong GL-H, Foucher J, Chan HL-Y, Le Bail B, et al. Diagnosis of fibrosis and cirrhosis using liver stiffness measurement in nonalcoholic fatty liver disease. Hepatology 2010; 51: 454-462. doi:10.1002/hep. 23312 
80. Castera L, Foucher J, Bernard P-H, Carvalho F, Allaix D, Merrouche W, et al. Pitfalls of liver stiffness measurement: a 5-year prospective study of 13,369 examinations. Hepatology 2010; 51 : 828-835. doi:10.1002/hep.23425

81. Cassinotto C, Boursier J, de Lédinghen V, et al. Liver stiffness in nonalcoholic fatty liver disease: a comparison of supersonic shear imaging, FibroScan, and ARFI with liver biopsy. Hepatology 2016; 63: 1817-1827.

82. Palmeri ML, et al. Noninvasive evaluation of hepatic fibrosis using acoustic radiation force based shear stiffness in patients with nonalcoholic fatty liver disease. J Hepatol 2011; 55: 666-672.

83. Xiao G, Zhu S, Xiao X, Yan L, Yang J, Wu G. Comparison of laboratory tests, ultrasound, or magnetic resonance elastography to detect fibrosis in patients with nonalcoholic fatty liver disease: A meta-analysis. Hepatology 2017; 66: 1486-1501. doi:10.1002/ hep. 29302.

84. Park CC, et al. Magnetic resonance elastography vs transient elastography in detection of fibrosis and noninvasive measurement of steatosis in patients with biopsy-proven nonalcoholic fatty liver disease. Gastroenterology 2017; 152: 598-607.

85. Loomba R, et al. Magnetic resonance elastography predicts advanced fibrosis in patients with nonalcoholic fatty liver disease: a prospective study. Hepatology 2014; 60: 1920-1928.

86. Rinella ME, Sanyal AJ. Management of NAFLD: A stage-based approach. Nat Rev Gastroenterol Hepatol 2016; 13: 196-205. doi:10.1038/nrgastro.2016.3

87. Chan WK, Nik Mustapha NR, Mahadeva S. A novel 2-step approach combining the NAFLD fibrosis score and liver stiffness measurement for predicting advanced fibrosis. Hepatol Int 2015; 9: 594-602. doi:10.1007/s12072-014-9596-7

88. Younossi ZM, Loomba R, Anstee QM, et al. Diagnostic modalities for non-alcoholic fatty liver disease (NAFLD), nonalcoholic steatohepatitis (NASH) and associated fibrosis. Hepatology 2018; 68: 349-360.

89. Stefan N, Häring HU, Cusi K. Non-alcoholic fatty liver disease: causes, diagnosis, cardiometabolic consequences, and treatment strategies. Lancet Diabetes Endocrinol 2019; 7: 313-324.

90. Khan R, Bril F, Newsome PN, Cusi K. Modulation of insulin resistance in NAFLD. Hepatology 2019; 70: 711-724.

91. Budd J, Cusi K. Non-alcoholic fatty liver disease: What does the primary care physician need to know? Am J Med 2020; 133: 536-543.

92. El-Agroudy NN, Kurzbach A, Rodionov RN, O'Sullivan J, Roden M, Birkenfeld AL, Pesta DH. Are lifestyle therapies effective for NAFLD treatment? Trends Endocrinol Metab 2019; 30: 701-709.

93. Parry SA, Hodson L. Managing NAFLD in type 2 diabetes: The effect of lifestyle interventions, a narrative review. Adv Ther 2020; 37: 1381-1406.

94. Lomonaco R, Sunny N, Bril F, Cusi K. Non-alcoholic fatty liver disease: Current issues and novel treatment approaches. Drugs 2013; 73: 1-14.

95. Corey KE, Rinella ME. Medical and surgical treatment options for nonalcoholic steatohepatitis. Dig Dis Sci 2016; 61: 1387-1397.

96. Belfort R, Harrison S, Brown K, et al. A placebo-controlled trial of pioglitazone in patients with non-alcoholic steatohepatitis (NASH). New Engl J Med 2006; 355: 2297-2307.

97. Cusi K, Orsak B, Bril F, et al. Long-term pioglitazone treatment for patients with NASH and prediabetes or type 2 diabetes mellitus: a randomized controlled trial. Ann Intern Med. 2016; 165: 305-315.
98. Bril F, Biernacki DM, Kalavalapalli S, et al. Role of vitamin E for nonalcoholic steatohepatitis in patients with type 2 diabetes: A randomized controlled trial. Diabetes Care 2019; 42: 1481-1488.

99. Aithal GP, Thomas JA, Kaye P V, et al. Randomized, placebo-controlled trial of pioglitazone in nondiabetic subjects with nonalcoholic steatohepatitis. Gastroenterology 2008; 135: 1176-1184.

100. Sanyal AJ, Chalasani N, Kowdley KV, et al. Pioglitazone, vitamin E, or placebo for nonalcoholic steatohepatitis. N Engl J Med 2010; 362: 1675-1685.

101. EASL. EASL-EASD-EASO clinical practice guidelines for the management of nonalcoholic fatty liver disease. J Hepatol 2016; 64: 1388-1402.

102. Mantovani A, Byrne Cd, Scorletti E, Mantzoros C, Targher G. Efficacy and safety of anti-hyperglycaemic drugs in patients with non-alcoholic fatty liver disease with or without diabetes: An updated systematic review of randomized controlled trials. Diabetes Metab 2020; S1262-3636(20)30002-1. doi: 10.1016/j. diabet.2019.12.007

103. DeFronzo RA, Tripathy D, Schwenke DC, et al. Pioglitazone for diabetes prevention in impaired glucose tolerance. Engl J Med 2011; 364: 1104-1115.

104. Dormandy JA, Charbonnel B, Eckland DJ, et al. Secondary prevention of macrovascular events in patients with type 2 diabetes in the PROactive Study (PROspective pioglitAzone Clinical Trial In macroVascular Events): a randomised controlled trial. The Lancet 2005; 366: 1279-1289.

105. Mazzone T, Meyer PM, Feinstein SB, et al. Effect of pioglitazone compared with glimepiride on carotid intima-media thickness in type 2 diabetes: a randomized trial. JAMA 2006; 296: 2572-2581.

106. Nissen SE, Nicholls SJ, Wolski K, et al. Comparison of pioglitazone vs. glimepiride on progression of coronary atherosclerosis in patients with type 2 diabetes: the PERISCOPE randomized controlled trial. JAMA 2008; 299: 1561-1573.

107. Kernan WN, Viscoli CM, Furie KL, et al. Pioglitazone after ischemic stroke or transient ischemic attack. N Engl J Med 2016; 374: 1321-1331.

108. Spence JD, Viscoli CM, Inzucchi SE, et al. Pioglitazone therapy in patients with stroke and prediabetes: A post hoc analysis of the IRIS randomized clinical trial. JAMA Neurol 2019; 76: 526-535.

109. Aronoff S, S Rosenblatt S, Braithwaite S, et al. Pioglitazone hydrochloride monotherapy improves glycemic control in the treatment of patients with type 2 diabetes: a 6-month randomized placebo-controlled dose-response study. The Pioglitazone 001 Study Group. Diabetes Care 2000; 23: 1605-1611.

110. Miyazaki Y, Mahankali A, Matsuda M, et al. Effect of pioglitazone on abdominal fat distribution and insulin sensitivity in type 2 diabetic patients. J Clin Endocrinol Metab 2002; 87: 2784-2791.

111. Clarke GD, Solis-Herrera C, Molina-Wilkins M, et al. Pioglitazone improves left ventricular diastolic function in subjects with diabetes. Diabetes Care 2017; 40: 1530-1536.

112. Yau H, Rivera K, Lomonaco R, Cusi K. The future of thiazolidinedione therapy in the management of type 2 diabetes mellitus. Curr Diab Rep 2013; 13: 329-341.

113. Zelniker TA, Wiviott SD, Raz I, et al. Comparison of the effects of glucagon-like peptide receptor agonists and sodium-glucose cotransporter 2 inhibitors for prevention of major adverse cardiovascular and renal outcomes in type 2 diabetes mellitus. Circulation 2019; 139: 2022-2031. 
114. Cusi K. Incretin-based therapies for the management of nonalcoholic fatty liver disease in patients with type 2 diabetes. Hepatology 2019; 69: 2318-2322.

115. Armstrong MJ, Gaunt P, Aithal GP, et al. Liraglutide safety and efficacy in patients with non-alcoholic steatohepatitis (LEAN): a multicentre, double-blind, randomised, placebo-controlled phase 2 study. Lancet 2016; 387: 679-690.

116. Gastaldelli A, Cusi K. From NASH to diabetes and from diabetes to NASH: Mechanisms and treatment options. JHEP Rep 2019; 1: 312-328.

117. Chilton RJ. Effects of sodium-glucose cotransporter-2 inhibitors on the cardiovascular and renal complications of type 2 diabetes. Diabetes Obes Metab 2020; 22: 16-29.

118. Eriksson JW, Lundkvist P, Jansson PA, et al. Effects of dapagliflozin and n-3 carboxylic acids on non-alcoholic fatty liver disease in people with type 2 diabetes: a double-blind randomised placebo-controlled study. Diabetologia 2018; 61: 1923-1934.

119. Latva-Rasku A, Honka MJ, Kullberg J, et al. The SGLT2 inhibitor dapagliflozin reduces liver fat but does not affect tissue insulin sensitivity: A randomized, double-blind, placebo-controlled study with 8-week treatment in type 2 diabetes patients. Diabetes Care 2019; 42: 931-937.

120. Cusi K, Bril F, Barb D, Polidori D, Sha S, Ghosh A, et al. Effect of canagliflozin treatment on hepatic triglyceride content and glucose metabolism in patients with type 2 diabetes. Diabetes Obes Metab 2019; 21: 812-821.

121. Kahl S, Gancheva S, Straßburger K, et al. Empagliflozin effectively lowers liver fat content in well-controlled type 2 diabetes: A randomized, double-blind, phase 4, placebo-controlled trial. Diabetes Care 2020; 43: 298-305.

122. Cusi K. Time to include nonalcoholic steatohepatitis in the management of patients with type 2 diabetes. Diabetes Care 2020; 43: $275-279$.
123. Gastaldelli A, Cusi K. From NASH to diabetes and from diabetes to NASH: Mechanisms and treatment options. JHEP Rep 2019; 1: 312-328.

124. Cusi K. A diabetologist's perspective of non-alcoholic steatohepatitis (NASH): Knowledge gaps and future directions. Liver Int 2020; 40 Suppl 1: 82-88.

125. Lavine JE, Schwimmer JB, Van Natta ML, Molleston JP, Murray KF, Rosenthal $\mathrm{P}$, et al. Effect of vitamin $\mathrm{E}$ or metformin for treatment of nonalcoholic fatty liver disease in children and adolescents: the TONIC randomized controlled trial. JAMA 2011; 305: 1659-1668.

126. Vilar-Gomez E, Vuppalanchi R, Gawrieh S, et al. Vitamin E improves transplant-free survival and hepatic decompensation among patients with nonalcoholic steatohepatitis and advanced fibrosis. Hepatology 2020; 71: 495-509.

127. Miller ER 3rd, Pastor-Barriuso R, et al. Meta-analysis: Highdosage vitamin E supplementation may increase all-cause mortality. Ann Intern Med 2005; 142 (1): 37-46.

128. Schurks M, Glynn RJ, Rist PM, Tzourio C, Kurth T. Effects of vitamin $\mathrm{E}$ on stroke subtypes: meta-analysis of randomised controlled trials [Internet]. BMJ. 2010; 341: c5702-c5702. Disponible en: http://dx.doi.org/10.1136/bmj.c5702.

129. Klein EA, Thompson IM Jr, Tangen CM, Crowley JJ, Lucia MS, Goodman PJ, et al. Vitamin E and the risk of prostate cancer: the Selenium and Vitamin E Cancer Prevention Trial (SELECT). JAMA 2011; 306: 1549-1556.

130. Wong RJ, Aguilar M, Cheung R, et al. Nonalcoholic steatohepatitis is the second leading etiology of liver disease among adults awaiting liver transplantation in the United States. Gastroenterology 2015; 148 (3): 547-555. 